Original Article

\title{
LIQUID CHROMATOGRAPHY TANDEM MASS SPECTROMETRY DETERMINATION METHOD OF BENCYCLOQUIDIUM BROMIDE: APPLICATION TO DRUG INTERACTION STUDY IN HUMAN
}

\author{
JANVIER ENGELBERT AGBOKPONTO1,2*, LOCONON ACHILLE YEMOA², ASSOGBA GABIN ASSANHOU², RUIJUAN \\ LIU $^{1}$, HABIB GANFON ${ }^{2}$, LI DING $^{*}$
}

${ }^{1}$ Department of Pharmaceutical Analysis, China Pharmaceutical University, Nanjing, China, ${ }^{2}$ UFR/Pharmacie, Faculte Des Sciences de la Sante, UAC, Cotonou, BENIN

*Email: aengelbert@yahoo.fr

Received: 27 Mar 2015, Revised and Accepted: 30 Aug 2021

\section{ABSTRACT}

Objective: This study was conducted to develop a sensitive and effective LC-MS/MS method for the determination of bencycloquidium bromide (BCQB) and its application in pharmacokinetic drug interaction study between BCQB and paroxetine.

Methods: The chromatographic separation was performed on Hedera ODS-2 C18 column with a mobile phase consisted of acetonitrile-10 mmol/l ammonium acetate containing $0.2 \%$ acetic acid $(33: 67, \mathrm{v} / \mathrm{v})$ at $550 \mu \mathrm{l} / \mathrm{min}$, and the plasma samples were processed using solid-phase extraction The MS/MS transitions were $m / z 330.2 \rightarrow 142.0$ for BCQB and $m / z 344.2 \rightarrow 156.1$ for the I. S in positive ESI mode.

Results: The validated method was linear over the concentration range of $2-1200 \mathrm{pg} / \mathrm{ml}$ with the correlation coefficient $r^{2}>0.998$. The intra-and inter-batch precisions of the assay were lower than $8.2 \%$ and $9.1 \%$, respectively. The lower limit of quantification (LLOQ) was $2 \mathrm{pg} / \mathrm{ml}$. The stability data at different storage conditions of $\mathrm{BCQB}$ were within $\pm 5 \% \mathrm{RE}$. The mean $A U C_{0-36}$ of $\mathrm{BCQB}$ was increased by approximately $33 \%$, after the administration of $\mathrm{BCQB}$ alone and upon co-administration with paroxetine during the drug interaction study.

Conclusion: The LC-MS/MS method validated in this study was robust, reproducible, accurate, precise and reliable and was successfully applied in the pharmacokinetic drug interaction studies.

Keywords: Bencycloquidium bromide, LC-MS/MS, Pharmacokinetic drug interaction, Paroxetine

(C) 2021 The Authors. Published by Innovare Academic Sciences Pvt Ltd. This is an open-access article under the CC BY license (https://creativecommons.org/licenses/by/4.0/) DOI: https://dx.doi.org/10.22159/ijpps.2021v13i10.5791. Journal homepage: https://innovareacademics.in/journals/index.php/ijpps.

\section{INTRODUCTION}

Bencycloquidium bromide (BCQB) is a selective muscarinic M1/M3 receptor antagonist developed for the treatment of rhinitis, asthma and chronic obstructive pulmonary disease (COPD) [1-3]. Studies on general pharmacological actions of BCQB suggested that it had no obvious effect on cardiovascular and respiratory in beagle dogs, and could decrease the extraction of the bronchus gland and inhibit the peristalsis of the small intestine in mice $[4,5]$. Under the clinical trial, an early study has shown its tolerability and efficacy [6]. However, the investigations of drug interactions of BCQB in humans remain an important part of its safety. Our recent study on in vitro metabolism and disposition of $\mathrm{BCQB}$ in human revealed that $\mathrm{BCQB}$ is the substrate of several metabolic enzymes. It is metabolized mainly by CYP2D6 and also weekly by CYP3A4/5 and CYP2C19 [7-8]. During BCQB drug development phase, it is required to perform pharmacokinetic drug interaction studies with other drugs (known as inhibitors or inducer of metabolic enzymes) based on blood concentration measurements. Most often, plasma is prepared and used for analytical investigation in such studies [9-10]. A number of bioanalytical methods have been described for the determination of BCQB in biological media [11-12]. Previous publications have described methods for the analysis of BCQB in animals' samples by LC-MS. However, the lower limit of quantitation (LLOQ) of the methods was too high (3 ng/ml and $0.05 \mathrm{ng} / \mathrm{ml}$ ) and not applicable. BCQB is an anticholinergic agent used in rhinitis, COPD and asthma, and is administered nasally at a very low dosage $(180 \mu \mathrm{g})$. Therefore a highly sensitive and selective determination method is required. An LC-MS method for the determination of BCQB in human plasma was reported [13], but was less sensitive for drug interactions study and required a long analytical run time.

In order to perform BCQB plasma measurements in connection with a clinical trial, the goal was to establish an analytical method with both high sensitivity and high capacity due to the expected low concentration of BCQB. We, therefore, designed and validated an effective LC-MS/MS method for BCQB determination. Our method was successfully applied in a pharmacokinetic drug interaction study between BCQB and paroxetine, a strong inhibitor of CYP2D6, and fulfilled the high demands of a low measuring range and a time constraint schedule.

\section{MATERIALS AND METHODS}

\section{Chemicals and reagents}

The reference standard of $\mathrm{BCOB}(>99.7 \%$ purity) and 1-ethylbencycloquidium bromide (IS) ( $>99.9 \%$ purity) (fig. 1) were supplied by Beijing Shiqiao Biological and Pharmaceutical Co. Ltd. (Beijing China). The weak cation-exchange solid-phase extraction (WCE-SPE) columns were purchased from Cleanert TM Angela Technologies (Beijing, China). Methanol and Acetonitrile were of HPLC grade and purchased from Merck KGaA (Darmstadt, Germany). Formic acid, acetic acid and ammonium acetate were of analytical grade and purchased from Nanjing Chemical Reagent Co., Ltd. (Nanjing, China). Distilled water was used throughout the experiment.
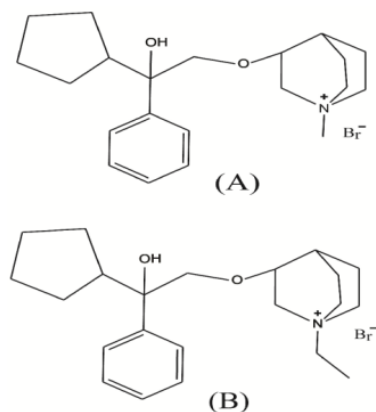

Fig. 1: Chemical structure of BCQB (A) and IS (B) 


\section{LC-MS/MS instrumentation and conditions}

The liquid chromatography was done on an Agilent 1200 Series liquid chromatography (Agilent Technologies, Palo Alto, CA, USA), which included an Agilent 1200 binary pump (model G1312B), vacuum degasser (model G1322A), Agilent 1200 autosampler (model G1367C), temperature-controlled column compartment (model G1330B). The chromatographic separation was achieved on a Hedera ODS-2 C18 column $(150 \mathrm{~mm} \times 2.1 \mathrm{~mm}$ i.d., $5 \mu \mathrm{m}$ d. p, Hanbon Sci. and Tech) protected by a security guard C18 column (4 $\mathrm{mm} \times 2.0 \mathrm{~mm}$ i.d., $5 \mu \mathrm{m}$ d. $\mathrm{p}$, Phenomenex, Torrance, CA, USA) with a mobile phase of acetonitrile- $10 \mathrm{mmol} / \mathrm{l}$ ammonium acetate solution containing $0.2 \%$ acetic acid $(33: 67, \mathrm{v} / \mathrm{v})$ at a flow rate of $550 \mu \mathrm{l} / \mathrm{min}$. The column temperature and auto-sampler temperature were maintained at $39{ }^{\circ} \mathrm{C}$ and $15{ }^{\circ} \mathrm{C}$, respectively. The injection volume was $10 \mu \mathrm{l}$. The LC system was coupled with an Agilent 6410B triple quadrupole mass spectrometry (Agilent Technologies, USA) equipped with an electrospray ionization source (model G1956B). The mass spectrometry was operated in positive ESI mode with the drying gas (N2) flow of $12 \mathrm{~L} / \mathrm{min}$, nebulizer pressure of $50 \mathrm{psi}$, drying gas temperature of $350{ }^{\circ} \mathrm{C}$, the capillary voltage of $4.5 \mathrm{kV}$. The fragmentation transitions for the multiple reaction monitoring (MRM) were $\mathrm{m} / \mathrm{z} 330.2 \rightarrow 142.0$ for BCQB and $\mathrm{m} / \mathrm{z} 344.2 \rightarrow 156.1$ for the IS (fig. 2). The fragmentor voltage value set for BCQB and the IS was $170 \mathrm{~V}$, and the collision energy was $40 \mathrm{eV}$.
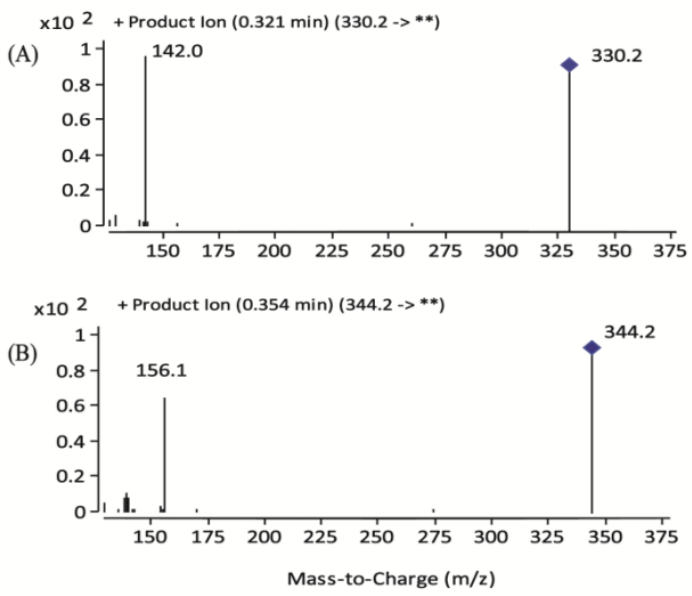

Fig. 2: Positive product ion mass spectra of BCQB (A) and IS (B)

\section{Preparation of calibration standards and quality control sample}

Calibration standards and quality control (QC) samples were prepared from independently weighed stocks. Standard stock solutions of BCQB and IS were prepared in methanol to a concentration of $1.006 \mathrm{mg} / \mathrm{ml}$ and $1.010 \mathrm{mg} / \mathrm{ml}$. The stock solution of BCQB was further serially diluted to yield working solutions at several concentration levels, 100.6, 48.29, 40.24, 32.19, 16.10, 4.829, $4.024,1.207,0.4024,0.2012,0.1408$ and $0.0804 \mathrm{ng} / \mathrm{ml}$. The working solution of I. S was prepared at the concentration of $6.05 \mathrm{ng} / \mathrm{ml}$ by diluting the standard stock solution in methanol. All stock and working solutions were kept at-20 ${ }^{\circ} \mathrm{C}$. Calibration standards and quality control samples were prepared by spiking blank plasma with the appropriate amount of working solutions. Calibration standards were of $2.01,5.03,10.06,30.18,100.6,402.4,804.8,1207 \mathrm{pg} / \mathrm{ml}$. QC samples were prepared at concentration levels of $3.52 \mathrm{pg} / \mathrm{ml}$ (Low), $120.7 \mathrm{pg} / \mathrm{ml}$ (Middle) and $1006 \mathrm{pg} / \mathrm{ml}$ (High).

\section{Sample preparation}

In this study, gravity was used to elute the solvents from the SPE column. The conditioning step involved activating the cartridge $(1$ $\mathrm{ml}$ ) with $2 \mathrm{ml}$ of methanol followed by $1 \mathrm{ml}$ of $\mathrm{H} 2 \mathrm{O}$ and then conditioning the cartridge with $2 \mathrm{ml}$ of ammonium acetate buffer ( $25 \mathrm{mmol}, \mathrm{pH}$ 7.0). Aliquot of $1 \mathrm{ml}$ plasma sample was transferred to a $2 \mathrm{ml}$ capped tube, into which $30 \mu \mathrm{l}$ of the I. S. solution $(6.05 \mathrm{ng} / \mathrm{ml})$ was spiked. The sample mixtures were vortexed for $30 \mathrm{~s}$ and centrifuged at $16,000 \mathrm{rpm}$ for $5 \mathrm{~min}$, and loaded onto the conditioned cartridge drop-wise. The lockstep involved treating the cartridge with $2 \mathrm{ml}$ of ammonium acetate buffer (25 mmol, pH 7.0) to adjust the $\mathrm{pH}$ of the system to ensure that the ionization of the sorbent bed and its ion-exchange interactions with the analytes were enabled. The wash step utilized $2 \mathrm{ml}$ of methanol to remove all unretained species from the sorbent bed. Elution was achieved by the addition of $2 \mathrm{ml}$ of methanol containing $2 \%$ formic acid. No vacuum system was employed in the SPE process. All plasma samples, condition and elution solutions were allowed to pass through the SPE cartridges with the aid of gravity to ensure that there was enough time for the equilibrium of ion-exchange reactions in every SPE step.

The eluted solution was transferred to a $10 \mathrm{ml}$ glass tube and evaporated to dryness at $40{ }^{\circ} \mathrm{C}$ under a stream of nitrogen. The dried extract was then dissolved in $100 \mathrm{ml}$ of the mobile phase. The reconstituted solution was transferred to an autosampler vial and an aliquot of $10 \mu \mathrm{l}$ injected into the chromatographic system.

\section{Method validation}

The method was validated according to the FDA's industrial guidance for the bioanalytical method validation [14]. The method was validated for selectivity, linearity, recovery, matrix effect (ME), precision, accuracy, and stability. Method sensitivity was determined by the limit of detection (LOD) and low limit of quantification (LLOQ) based on a signal-to-noise ratio of 3:1 and 7:1, respectively. Selectivity and matrix effect was assessed by comparing the chromatograms of six different batches of blank plasma obtained from six different sources with those of corresponding spiked plasma. Each blank sample was tested for no endogenous interferences. Extraction recovery in plasma matrix for BCQB was determined at the three levels of $\mathrm{QC}$ and calculated as the ratio of analyte peak area from extracted QC samples to that from extracted blank matrices spiked with BCQB standard solution. To assess carryover effects, blanks were injected immediately after the highest calibration standard (ULOQ), and the response of any interfering peak had to be less than $20 \%$ of the response of an LLOQ sample. All validation experiments were carried out at three QC levels (Low QC, Middle QC and High QC) for precision and accuracy assessment. The precisions and accuracies should be within $\pm 15 \%$ except for LLOQ level, which should be within $\pm 20 \%$. Auto-sampler stability, benchtop stability, freeze-thaw stability, post preparative stability and long-term stability of the analyte were assessed. Samples were considered to be stable when their relative error (RE) was less than $15 \%$.

\section{Pharmacokinetic study}

The proposed analytical method was applied to the study of pharmacokinetic drug interaction between BCQB and paroxetine. The clinical pharmacokinetic trial was performed in accordance with guidelines established by the Declaration of Helsinki. The Ethics Committee of the West China Hospital of Sichuan University approved the study protocol and written informed consent had been obtained from each participant. The design of the study was an open-label, single-dose, two periods, single sequence crossover study. Chinese healthy volunteers were administered nasally a single dose of $180 \mu \mathrm{g}$ of BCQB alone on day 1. Blood samples for pharmacokinetic evaluation were collected at 0 (pre-dose), $2 \mathrm{~min}, 5$ min, 10 min, 15 min, 30 min, 1 h, 2 h, 4 h, 6 h, 9 h, 12 h, 15 h, 24 h and $36 \mathrm{~h}$ after BCQB administration. After a $7 \mathrm{~d}$ wash-out period, subjects were administered $20 \mathrm{mg}$ of paroxetine once a day from day 8 to day 17 and $180 \mu \mathrm{g}$ of BCQB were co-administered with $20 \mathrm{mg}$ of paroxetine on day 18. Blood samples were collected as mentioned above. The plasma fractions were stored at $-20^{\circ} \mathrm{C}$ until analysis. An aliquot of $1 \mathrm{ml}$ of thawed plasma samples was spiked with the IS and processed as mentioned in Section 2.4 above. Each analytical run was validated according to the FDA guidance for industry: Bioanalytical and method validation.

\section{RESULTS AND DISCUSSION}

\section{Method development and optimization}

Chromatographic conditions, especially the composition of the mobile phase, were optimized to enhance the sensitivity and achieve 
better separation from an endogenous matrix, as well as shorten the total run time. In this study, acetonitrile rather than methanol was chosen as organic eluent because it provided a higher mass spectrometric response and lower background noise. Acetonitrile had a strong eluting strength on endogenous compounds, eliminated matrix effect and shortened the total analytical run time to $7.5 \mathrm{~min}$ instead of $12 \mathrm{~min}$ in previous methods. The LLOQ and ULOQ were improved and the calibration range was extended $(2-1200 \mathrm{pg} / \mathrm{ml})$ to fit into the drug drugs interaction study without further dilution step. The mass spectrometry valve was diverted to waste from 5.5 min to 7.5 min when impurities from endogenous compounds were eluting from the HPLC.

\section{Method validation}

\section{Selectivity and carryover}

The selectivity of the method was determined by analyzing blank controls from six different individuals. There was no endogenous interference observed at the retention times of BCQB and the IS, which were 3.35 and 4.25 , respectively. No carry-over peaks were observed at the retention times of BCQB and the IS in the chromatogram of blank plasma injected after the highest concentration (ULOQ) sample. Typical MRM chromatograms of blank plasma samples, blank plasma spiked with BCQB at LLOQ level and the internal standard, and a plasma sample from a healthy volunteer at $1 \mathrm{~h}$ after dosing, respectively, are shown in fig. 3 .

\section{Linearity, accuracy and precision}

The Calibration curves were linear over the concentration range of $2-1200 \mathrm{pg} / \mathrm{ml}$ with a correlation coefficient $r^{2}>0.998$. The typical equations of the calibration curves were $f=0.006380 \times C+0.01538$, where $f$ represents the peak area ratio of the analytes to the IS and $C$ represents the concentration of the analytes. Validation samples of five replicates of the LLOQ and QC samples were prepared and analyzed in three separate analytical batches to evaluate the accuracy and intra-day and inter-day precision of the methods. Assay precision was calculated using the relative standard deviation (R. SD \%) and performing a one-way analysis of variance (ANOVA). The precision (RSD) and accuracy (RE) for quantification of BCQB in human plasma are summarized in table 1 . The results showed that both intra-day and inter-day values were all within the acceptable ranges. The method was accurate and precise.

\section{Matrix effect and recovery}

The matrix effects evaluated for the QC standards at three concentration levels ranged from $98.3 \%$ to $103.3 \%$ for $\mathrm{BCQB}$, suggesting no ion suppression or enhancement from the plasma matrix to the analytes. The mean recoveries, mean (RSD), of analytes at three concentration levels $(3.52,120.7$, and $1006 \mathrm{pg} / \mathrm{ml})$ were $88.2 \%$ (4.7\%), $85.3 \%$ (7.0\%) and $87.2 \%$ (2.8\%) for BCQB, respectively. The Matrix effect and recovery of the IS were $104.0 \%$ and $89.5 \%(3.0 \%)$, respectively.
(A)

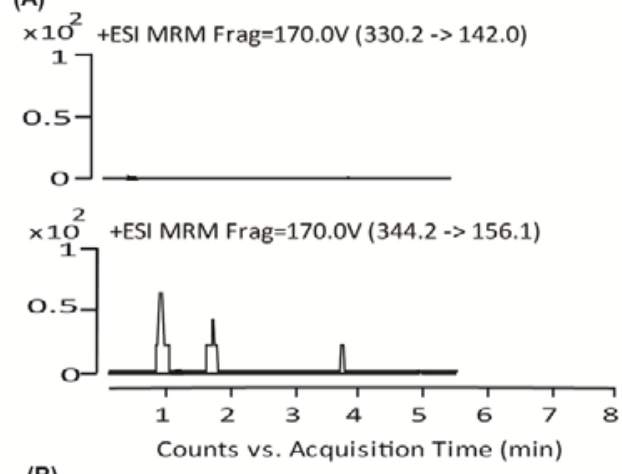

(B)

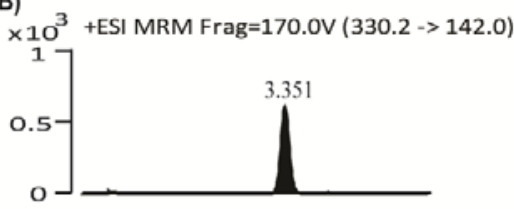

$\times 10^{2}+$ ESI MRM Frag=170.0V (344.2 -> 156.1)

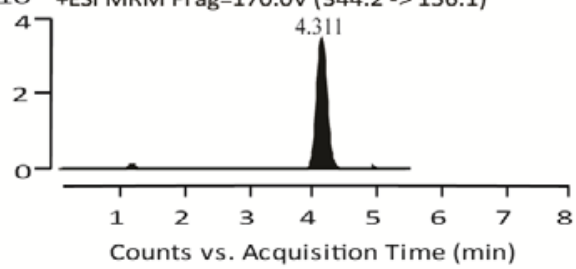

(C)

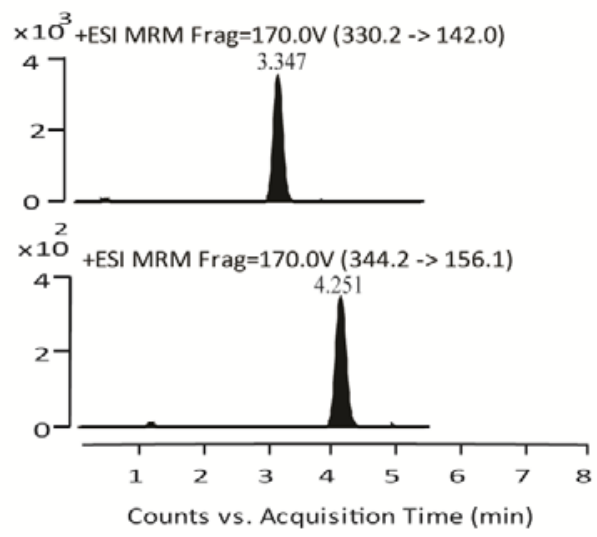

Fig. 3: Typical MRM chromatograms of BCQB and IS. Blank plasma sample (A), blank plasma sample spiked with the analytes at LLOQ and IS (B), and plasma sample from a healthy volunteer at $1 \mathrm{~h}$ after dosing (C)

Table 1: Precision and accuracy data for the analysis of BCQB in human plasma (n =5)

\begin{tabular}{llllll}
\hline Analytes & \multicolumn{2}{l}{ Concentration level $(\mathbf{p g} / \mathbf{m l})$} & RSD (\%) & & RE (\%) \\
\cline { 2 - 5 } & Added & Measured & Intra-batch & Inter-batch & 3.0 \\
Low QC & 3.520 & $3.652 \pm 0.3$ & 4.6 & 9.1 & -1.3 \\
Middle QC & 120.7 & $119.1 \pm 5.5$ & 4.5 & 6.2 & -0.9 \\
High QC & 1006 & $997.1 \pm 45.1$ & & \\
\hline
\end{tabular}

Table 2: Stability of BCQB in human plasma under various storage conditions $(n=3)$

\begin{tabular}{|c|c|c|c|c|}
\hline \multirow[t]{2}{*}{ Storage conditions } & \multicolumn{2}{|l|}{$10.06 \mathrm{pg} / \mathrm{ml}$} & \multicolumn{2}{|l|}{$1006 \mathrm{pg} / \mathrm{ml}$} \\
\hline & mean $\pm S D$ & $R E(\%)$ & mean \pm SD & $R E(\%)$ \\
\hline Room temperature, $15 \mathrm{~h}$ & $4.034 \pm 0.11$ & 12.4 & $1012 \pm 25$ & -1.4 \\
\hline Auto-sampler stability, $14 \mathrm{~h}$ & $3.733 \pm 0.57$ & 4.0 & $983.1 \pm 29.7$ & -4.2 \\
\hline Post-preparative stability, $48 \mathrm{~h}$ & $3.716 \pm 0.41$ & 3.5 & $1030 \pm 21$ & 0.4 \\
\hline Three freeze/thaw cycles & $3.852 \pm 0.25$ & 7.4 & $1028 \pm 10$ & 0.2 \\
\hline Long term stability, $15 \mathrm{w}$ & $3.747 \pm 0.48$ & 4.4 & $1076 \pm 14$ & 4.9 \\
\hline
\end{tabular}




\section{Stability studies}

The stability results summarized in table 2 revealed that BCQB was stable in the plasma sample for $15 \mathrm{~h}$ at room temperature and after three freeze-thaw cycles of $48 \mathrm{~h}$. The analytes in processed plasma samples were stable in the auto-sampler for $14 \mathrm{~h}$ at $15{ }^{\circ} \mathrm{C}$. The postpreparative stability was accessed for $48 \mathrm{~h}$. The spiked plasma samples with $\mathrm{BCQB}$ stored at- $20{ }^{\circ} \mathrm{C}$ for long-term stability were found stable for a minimum period of $15 \mathrm{w}$.

\section{Application}

The validated LC-MS/MS method was successfully applied to the pharmacokinetic drug interaction studies of BCQB in twenty-one healthy Chinese volunteers [15]. The pharmacokinetic results of $\mathrm{BCQB}$ alone and BCQB co-administered with paroxetine are presented in fig. 4. Absorption of BCQB after nasal administration was rapid with a median $T_{\max }$ of $10 \mathrm{~min}$ in both groups. The plasma concentrations decreased in a biphasic manner with the mean $t_{1 / 2}$ of approximately 10 to $11 \mathrm{~h}$ across the doses. The co-administration of paroxetine with $\mathrm{BCQB}$ decreased the elimination rate of $\mathrm{BCQB}$ and increased its plasma concentration at the terminal phase. The geometric mean $A U C_{0-36}$ of $\mathrm{BCQB}$ was increased by approximately $33 \%$ during concomitant administration with paroxetine. The mean $C_{\max }$ and $t_{1 / 2}$ data showed no statistically significant difference $(p>0.05)$. The results showed large inter-individual variability in BCQB plasma concentration; this was shown by the mean $C_{\max }$ data for both treatments $240.1 \pm 186.8 \mathrm{pg} / \mathrm{ml}$ and $247.3 \pm 235.9 \mathrm{pg} / \mathrm{ml}$, respectively.

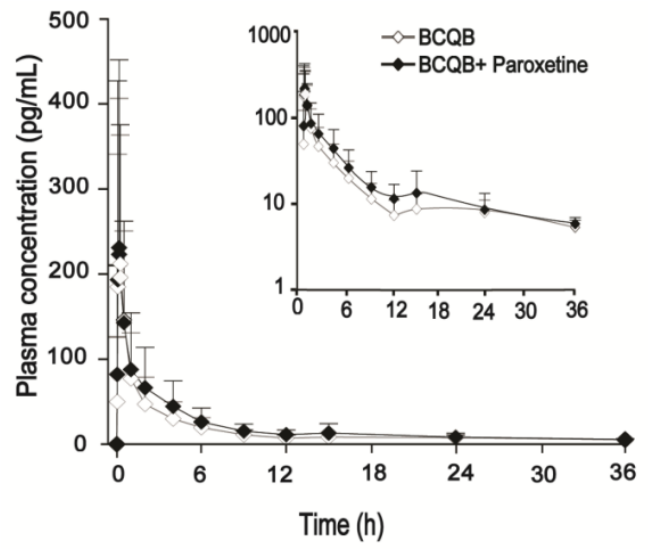

Fig. 4: Mean plasma concentration-time profiles of BCQB after single-dose administration and co-administration with paroxetine in healthy Chinese subjects, respectively [15]

\section{CONCLUSION}

A new sensitive, accurate, selective, and simple LC-ESI-MS/MS method has been developed and validated for the quantitative determination of BCQB in human plasma. The LC-MS/MS method validated in this study was robust, reproducible, accurate, precise and reliable. The method was successfully applied for the pharmacokinetic drug interaction studies between BCQB and other drugs.

\section{CONFLICT OF INTERESTS}

The authors have declared no conflict of interest.

\section{REFERENCES}

1. Cao R, Dong XW, Jiang JX, Yan XF, He JS, Deng YM, Li FF, Bao MJ, Xie YC, Chen XP, Xie QM. M(3) muscarinic receptor antagonist bencycloquidium bromide attenuates allergic airway inflammation, hyperresponsiveness and remodeling in mice.
Eur J Pharmacol. 2011;655(1-3):83-90. doi: 10.1016/ j.ejphar.2011.01.024, PMID 21277298.

2. Jiang JX, Cao R, Deng WD, Jin F, Dong XW, Zhu Y, Chen XP, Xie YC, Bao MJ, Li FF, Xie QM. Characterization of bencycloquidium bromide, a novel muscarinic $\mathrm{M}(3)$ receptor antagonist in guinea pig airways. Eur J Pharmacol. 2011;655(1-3):74-82. doi: 10.1016/j.ejphar.2011.01.017, PMID 21272572.

3. Li J, He H, Zhou YD, Yuan P, Chen XP. Subchronic toxicity and toxicokinetics of long-term intranasal administration of bencycloquidium bromide: a 91-day study in dogs. Regul Toxicol Pharmacol. 2011;59(2):343-52. doi: 10.1016/j.yrtph.2010.11.006, PMID 21130130.

4. Li J, Zhou YD, Chen XP. Preliminary observation on the antiinflammatory action and anti-pruritic action of bencycloquidium bromide. Chin J New Drugs. 2007;16:1182-4.

5. Li J, Zhou YD, Chen XP. Experimental study on general pharmacological actions of bencycloquidium bromide. J Chongqing Med Univ. 2007;32:506-10.

6. Sun L, Ding L, Wang Y, Zhou W, Yan Z, Sun W, Zhang H, Ou N, Chen X. Pharmacokinetics, safety and tolerability of bencycloquidium bromide, a novel selective muscarinic M1/M3 receptor antagonist, after single and multiple intranasal doses in healthy Chinese subjects. Drugs RD. 2012;12(1):17-28. doi: 10.2165/11599330-000000000-00000, PMID 22339483.

7. Agbokponto JE, Zhang L, Hu L, Feng H, Ding L. In vitro metabolism of Bencycloquidium bromide and its inhibitory effects on human P450 isoenzymes: implication of CYP2D6, CYP2C19 and CYP3A4/5. Eur J Drug Metab Pharmacokinet. 2016;41(1):69-77. doi: 10.1007/s13318-014-0237-2, PMID 25425116.

8. Sun L, Ding L, Yan Z, Du XL, Luo XM, Chen X. Determination of the inhibitory potency of bencycloquidium bromide on rat liver cytochrome P450 by LC-MS/MS. J China Pharm Univ. 2013;44(2):134-40.

9. Shankar GG, Pragney D, Ramakrishna S. Simultaneous determination of atorvastatin and olmesartan medoxomil in rat plasma by liquid chromatography-electrospray ionization tandem mass spectrometry and its application to pharmacokinetics in rats. Int $J$ Pharm Pharm Sci. 2014;6(10):464-8.

10. Hu L, Agbokponto JE, Li X, Ding L, Liu B, Zhong S, Zhang X, Du Y. In vivo and in vitro evidence of the sex-dependent pharmacokinetics and disposition of G004, a potential hypoglycemic agent, in rats. Eur J Drug Metab Pharmacokinet. 2015;40(2):187-202. doi: 10.1007/s13318-014-0196-7, PMID 24696324.

11. Xu Q, Ding L, Liu WY, Bian XJ, Tang W. Determination of bencycloquidium bromide in rat plasma by liquid chromatography-electrospray ionization-mass spectrometry. J Chromatogr B Analyt Technol Biomed Life Sci. 2007;846(12):209-14. doi: 10.1016/j.jchromb.2006.09.028, PMID 17055347.

12. Jiang B, Ruan Z, Lou H, Dong X, Xie Q. Determination of bencycloquidium bromide in dog plasma by liquid chromatography with electrospray ionization tandem mass spectrometry. Biomed Chromatogr. 2010;24(5):490-6. doi: 10.1002/bmc.1316, PMID 19688815.

13. Zhou W, Ding L, Wang Y, Sun L, Huang Y, Hu L, Chen X. Solid phase extraction and liquid chromatography-electrospray ionization-mass spectrometry for the determination of bencycloquidium bromide in human plasma. J Chromatogr B Analyt Technol Biomed Life Sci. 2009;877(10):897-901. doi: 10.1016/j.jchromb.2009.02.027, PMID 19251489.

14. International Conference on Harmonization (ICH), Guidance for Industry: Bioanalytical Method Validation. Register, Rockville: US FDA; 2001.

15. Agbokponto JE, Luo Z, Liu R, Liu Z, Liang M, Ding L. Study of pharmacokinetic interaction of paroxetine and roxithromycin on bencycloquidium bromide in healthy subjects. Eur J Pharm Sci. 2015;69:37-43. doi: 10.1016/j.ejps.2014.12.019, PMID 25559065. 